\title{
Bilateral squamosal synostosis: unusual presentation of chromosome 1p12-1p13.3 deletion. Illustrative case
}

\author{
Sarut Chaisrisawadisuk, MD, FRCST, ${ }^{1,4}$ Nithiwat Vatanavicharn, MD, ${ }^{2}$ Verayuth Praphanphoj, MD, ${ }^{3}$ \\ Peter J. Anderson, DSc, FRACS, ${ }^{4,5}$ and Mark H. Moore, MBChB, FRACS ${ }^{4}$ \\ ${ }^{1}$ Division of Plastic Surgery, Department of Surgery, and 2Division of Medical Genetics, Department of Paediatrics, Faculty of Medicine Siriraj Hospital, Mahidol University, \\ Bangkok, Thailand; ${ }^{3}$ Medical Genetics Center, Sai Mai District, Bangkok, Thailand; ${ }^{4} \mathrm{Cleft}$ and Craniofacial South Australia, Women's and Children's Hospital, North Adelaide, \\ South Australia, Australia; and ${ }^{5}$ Faculty of Health and Medical Sciences, University of Adelaide, Adelaide, South Australia, Australia
}

BACKGROUND Squamosal sutures are minor sutures of the human skull. Early isolated fusion of the sutures (squamosal synostosis) is rarely found. OBSERVATIONS The authors report a case of a girl who presented with an abnormal head shape and bilateral squamosal synostosis. Genetic testing revealed a chromosome 1p12-1p13.3 deletion. She has been managed with conservative treatment of the synostosis. She has global developmental delay and multiple anomalies due to the chromosome abnormality.

LESSONS Isolated squamosal suture synostosis could be an uncommon feature of chromosome 1p12-1p13.3 deletion.

https://thejns.org/doi/abs/10.3171/CASE20102

KEYWORDS cranial sutures; craniosynostosis; chromosome 1p12-1p13.3 deletion

The squamosal suture, one of the minor sutures of the human calvaria, sits between the temporal and parietal bones and extends from the pterion, curving around the temporal squama. ${ }^{1}$ The suture is composed of a sphenosquamosal part (vertically oriented) and a temporosquamosal (horizontally oriented) part; ${ }^{2}$ their physiological fusion has been reported to normally start in the late $30 \mathrm{~s}^{3}$ In craniofacial patients, isolated squamosal synostosis-a condition in which there is early fusion of the squamosal sutures-is found in rare instances. ${ }^{4-6}$ There have been numerous reports of this synostosis being associated with some syndromes. ${ }^{4-11}$ We previously reported a case of late presentation of isolated bilateral squamosal synostosis. ${ }^{9}$ We now report a case of a 4-year-old girl who presented at 1 year of age with an abnormal head shape secondary to bilateral squamosal synostosis, and she also had chromosome 1p12-1p13.3 deletion. She has been managed with close monitoring. She continues her neurological development, albeit at a slow rate.

\section{Illustrative Case}

A 12-month-old girl presented with widening of the biparietal areas of the head (Fig. 1). She was born to nonconsanguineous parents with no familial history of congenital anomalies. An amniocentesis revealed chromosome $46, X X$. She was born at 38 weeks of gestation. Her physical examination at 1 year of age showed a widening of both parietal bones and an open anterior fontanelle. The finding of her fundus examination was unremarkable. Dysmorphology examination demonstrated upward-slanting eyes with exotropia, long philtrum, thin upper lip, a single transverse palmar crease on the right hand, and postaxial polydactyly of the left foot. Echocardiography showed mild coarctation of the aorta and a hypoplastic aortic arch. She had global developmental delay and failure to thrive, with her height and weight under the third percentile. Three-dimensional computed tomography (3D-CT) showed bilateral squamosal suture fusion with a $4.6 \times 4.6-\mathrm{cm}$ anterior fontanelle (Fig. 2). Ventriculomegaly was not observed.

Mutation analysis of the fibroblast growth factor receptor 2 (FGFR2) gene did not show any pathogenic variant. A blood karyotype study revealed $46, \mathrm{XX}$. We further conducted a chromosome microarray using an Affymetrix Cyto $750 \mathrm{~K}$ array, which revealed a 10.5-Mb deletion on chromosome 1p12-1p13.3 (chr1: 109,461,754-119,984,872) (Fig. 3). The deleted chromosome region of our patient contained 148 genes (Table 1), of which 95 were listed in the Online Mendelian Inheritance in Man database. Only 30

ABBREVIATIONS 3D-CT = three-dimensional computed tomography; FGFR2 = fibroblast growth factor receptor 2 ; HMG = hydroxymethylglutaryl INCLUDE WHEN CITING Published January 18, 2021; DOI: 10.3171/CASE20102.

SUBMITTED November 9, 2020. ACCEPTED November 19, 2020.

(c) 2021 The authors, CC BY-NC-ND 4.0 (http://creativecommons.org/licenses/by-nc-nd/4.0/). 


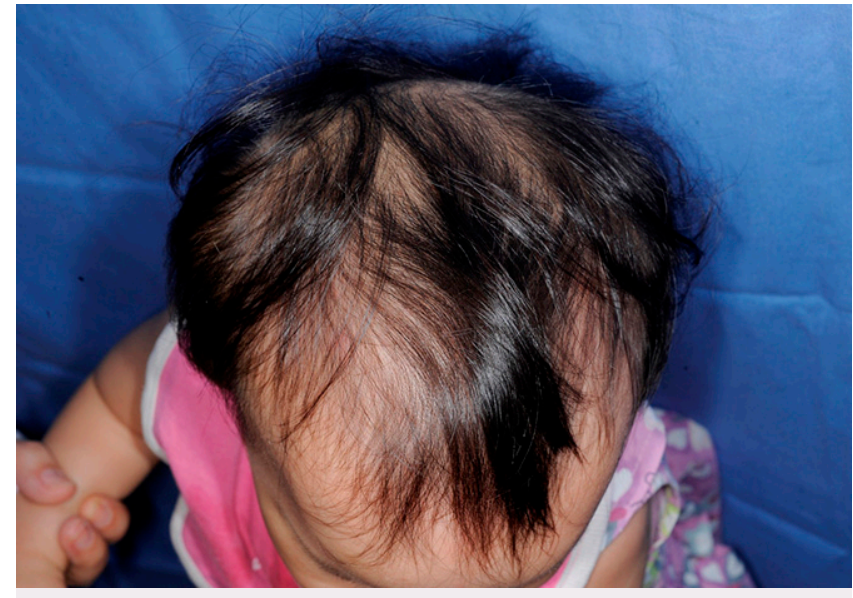

FIG. 1. Abnormal head shape, with bilateral bulging of the parietal regions.

of these genes were known to be associated with Mendelian disease phenotypes (Table 2). The karyotype studies of the parents revealed balanced chromosome insertion 46,XY,ins(18;1)(p11.2; $\mathrm{p} 13.1 \mathrm{p} 13.3)$ in the father and normal chromosome in the mother. The recurrence risk of unbalanced chromosome aberrations was discussed with the family.

After a multidisciplinary discussion, the risks and benefits of transcranial surgery were acknowledged. The parents decided on conservative treatment for the bilateral squamosal synostosis. Coarctation of the aorta was successfully treated by a balloon angioplasty when the patient was 2 years of age. The patient is currently 4 years old and undergoing regular follow-up. There is no sign of increased intracranial pressure. Despite some progression, her neurological development is still delayed.

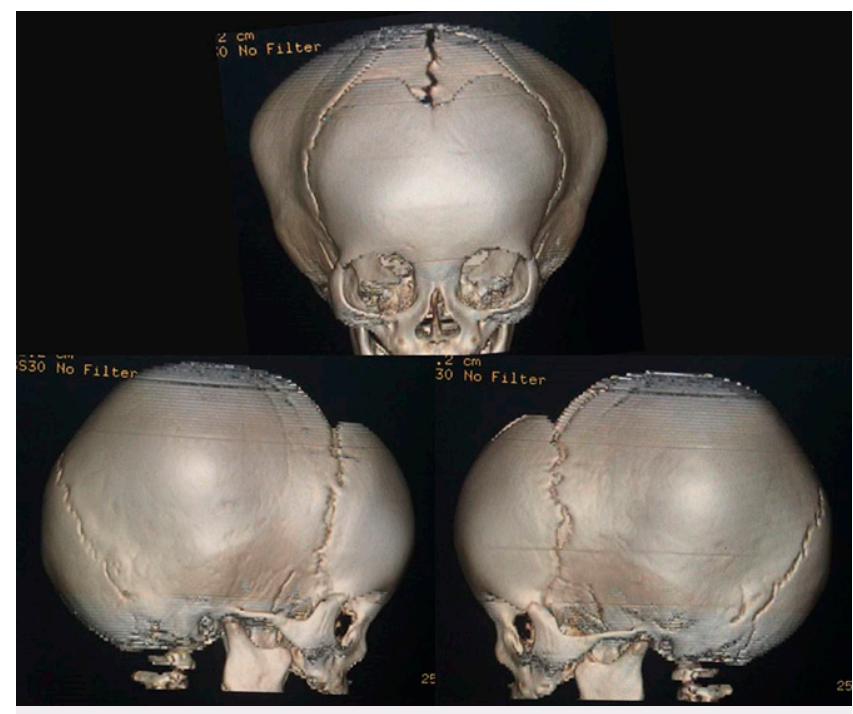

FIG. 2. 3D-CT scans showing the fusion at both the parietosquamosal and parietomastoid sutures, with bulging of both parietal bones. Widening of the anterior fontanelle $(4.6 \times 4.6 \mathrm{~cm})$ is present.
TABLE 1. List of 148 genes in the deleted chromosome region

SYPL2, ATXN7L2, CYB561D1, AMIG01, GPR61, GNAI3, MIR197, GNAT2, AMPD2, GSTM4, GSTM2, GSTM1, GSTM5, GSTM3, EPS8L3, CSF1, AHCYL1, STRIP1, ALX3, LINC01397, UBL4B, SLC6A17, KCNC4-AS1, KCNC4, LOC440600, RBM15, SLC16A4, LAMTOR5, LAMTOR5-AS1, PROK1, CYMP, LOC440602, KCNA10, KCNA2, KCNA3, CD53, LRIF1, DRAM2, CEPT1, DENND2D, CHI3L2, CHIAP2, CHIA, PIFO, PGCP1, OVGP1, WDRR77, ATP5F1, C1orf162, TMIGD3, ADORA3, LINC01160, RAP1A, FAM212B, FAM212B-AS1,

LOC101928718, DDX20, KCND3, KCND3-IT1, KCND3-AS1, LOC643355, CTTNBP2NL, MIR4256, WNT2B, ST7L, CAPZA1, MOV10, RHOC, PPM1J, FAM19A3, LINC01356, SLC16A1, AKR7A2P1, SLC16A1-AS1, LOC100996251, LRIG2, LOC643441, MAG/3, PHTF1, RSBN1, AP4B1-AS1, PRPN22, BLC2L15, AP4B1, DCLRE1B, HIPK1AS1, HIPK1, OLFML3, SYT6, TRIM33, BCAS2, DENND2C, AMPD1, NRAS, CSDE1, SIKE1, SYCP1, TSHB, TSPAN2, NGF, VANGL1, CASQ2, NHLH2, LOC101928995, LOC101928977, SLC22A15, MAB21L3, ATP1A1, ATP1A1-AS1, LOC101929023, CD58, MIR548AC, IGSF3, MIR320B1, C1orf137, CD2, PTGFRN, CD101, LOC101929099, TTF2, MIR942, TRIM45, VTCN1, LINC01525, MAN1A2,

LOC100996263, FAM46C, GDAP2, WDR3, SPAG17, TBX15, LOC105378933, WARS2, WARS2-IT1, LOC101929147, HAO2, HAO2IT1, HSD3B2, HSD3B1, HSD3B4P, LINC00622, ZNF697, PHGDH, HMGCS2, REG4, NBPF7, ADAM30, NOTCH2

\section{Discussion}

\section{Observations}

We present an isolated case of a patient with bilateral squamosal suture synostosis with chromosome 1p12-1p13.3 deletion. A spectrum of unpatterned skull shapes (plagiocephaly, brachycephaly, and scaphocephaly) has been reported to be linked with this synostosis..$^{1,5,7}$ In 2008, Greene et al. reported finding 1 case of isolated bilateral frontosphenoidal-bilateral squamosal suture synostosis among 518 patients in their database. ${ }^{6}$ In 2010, Ranger et al. ${ }^{1}$ presented 2 cases of squamosal synostosis. One was a nonsyndromic patient with unilateral squamosal synostosis, and the other was a patient with Crouzon syndrome with multiple synostoses, including bilateral squamosal sutures. Both patients, however, had good neurodevelopmental outcomes following extensive cranial vault remodeling. ${ }^{1}$ In 2012, Smartt et al. ${ }^{7}$ described 14 patients with squamosal synostosis with variations in disease presentation and treatment. Those researchers proposed that conservative treatment should be used for the initial management of isolated squamosal synostosis cases, such as close observation or the performance of helmet molding. In addition, they recommended that cranial vault surgery should be reserved for patients with an elevated intracranial pressure or substantial cranial asymmetry. ${ }^{7} \mathrm{~A}$ study by Eley et al. also showed success in the conservative management of 5 cases of isolated squamosal synostosis. ${ }^{8}$ That approach corresponds with our earlier report in which we proposed that surgical intervention is indicated in the presence of either a significant craniofacial deformity or raised intracranial hypertension contributing to neurodevelopmental delay. ${ }^{9}$

No clear etiology has been identified as being associated with isolated bilateral squamosal synostosis. The condition has been reported in cases of syndromic craniosynostosis, including Crouzon, Pfeiffer, and Muenke syndromes. , $^{1,8,8,10,11}$ A case report of 

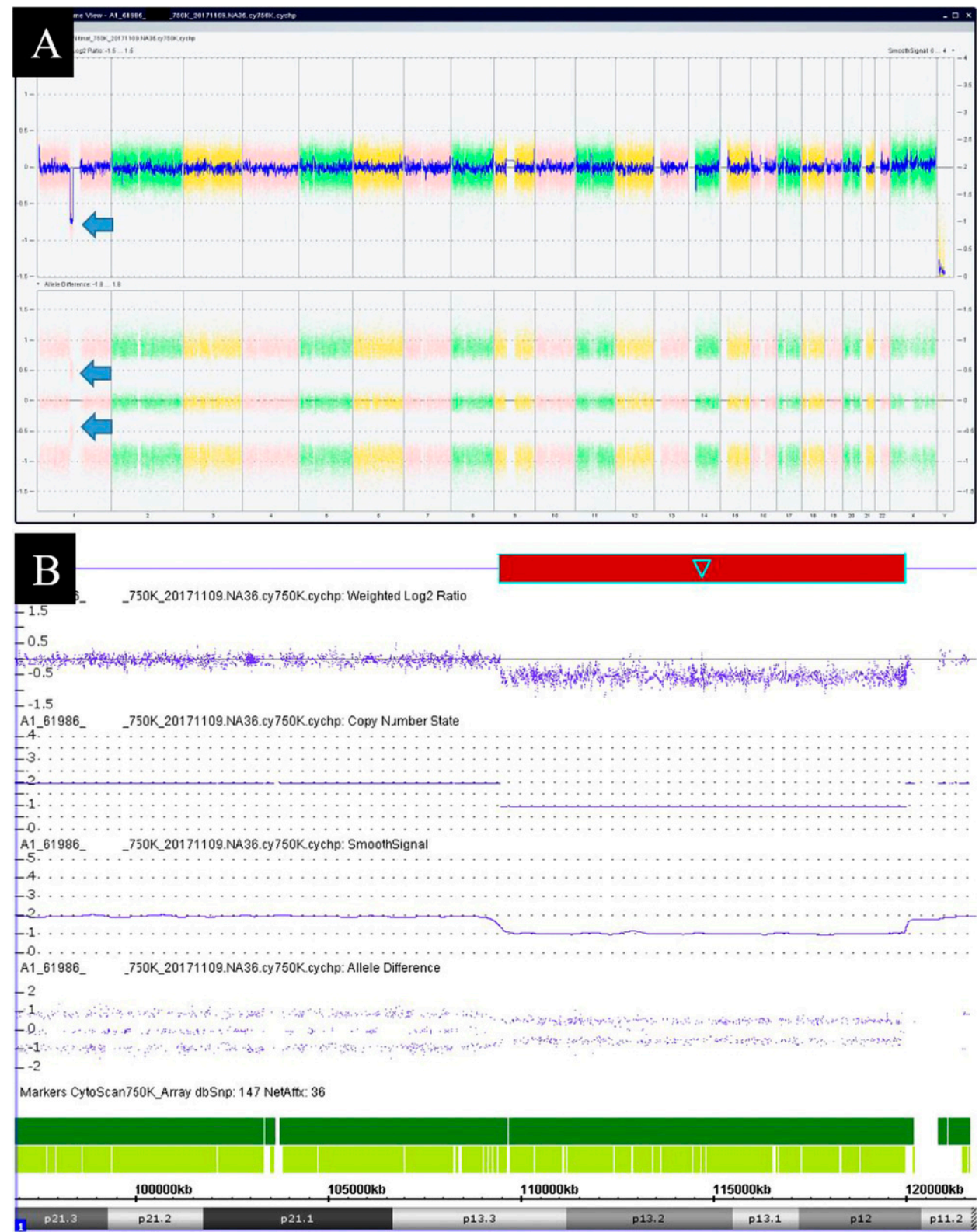

FIG. 3. A: Whole-genome copy number variation and single-nucleotide polymorphism (SNP) views demonstrated copy number lost on chromosome 1 (arrows). B: 10.5-Mb heterozygous deletion on chromosome 1p12-1p13.3 confirmed by copy number probes (upper 3 tracks) and SNP probes (bottom track).

a patient with Muenke syndrome with bilateral squamosal synostosis showed bilateral temporosquamosal and parietomastoid suture fusion, whereas the sphenosquamosal sutures were patent, on 3D-CT scans. ${ }^{10}$ Those findings are relevant to our case presentation. Runyan et al. found that patients with an FGFR2 mutation had significantly higher synostosis of the squamosal arch (odds ratio 2.73). ${ }^{12}$ However, an FGFR2 pathogenic variant was not identified in our reported case. Among the genes in the deleted chromosome region in our patient and known to cause Mendelian disease phenotypes, some have been reported to be related to craniofacial development. Twigg et al. identified mutations in $A L X 3$ encoding aristaless-related ALX homeobox 3 transcription factor in 7 families with frontonasal dysplasia; craniosynostosis, however, was not mentioned. ${ }^{13}$ Narro-Donate et al. ${ }^{14}$ reported a case of a 30-month-old girl who had been diagnosed with Alagille syndrome and a unicoronal synostosis. The Alagille syndrome was a multisystem autosomal dominant disorder caused by NOTCH2 mutations. ${ }^{14}$ Another study showed a correlation between TWIST1 and NOTCH2. Although TWIST1 positively regulates JAGGED1 in sutural mesenchyme, JAGGED1 represses NOTCH2. JAGGED1/ $\mathrm{NOTCH}$ functions in the initial specification of sutural cells and in the boundary between the osteogenic and nonosteogenic 
TABLE 2. List of 30 deleted genes in our patient associated with Mendelian disease phenotypes in the OMIM database

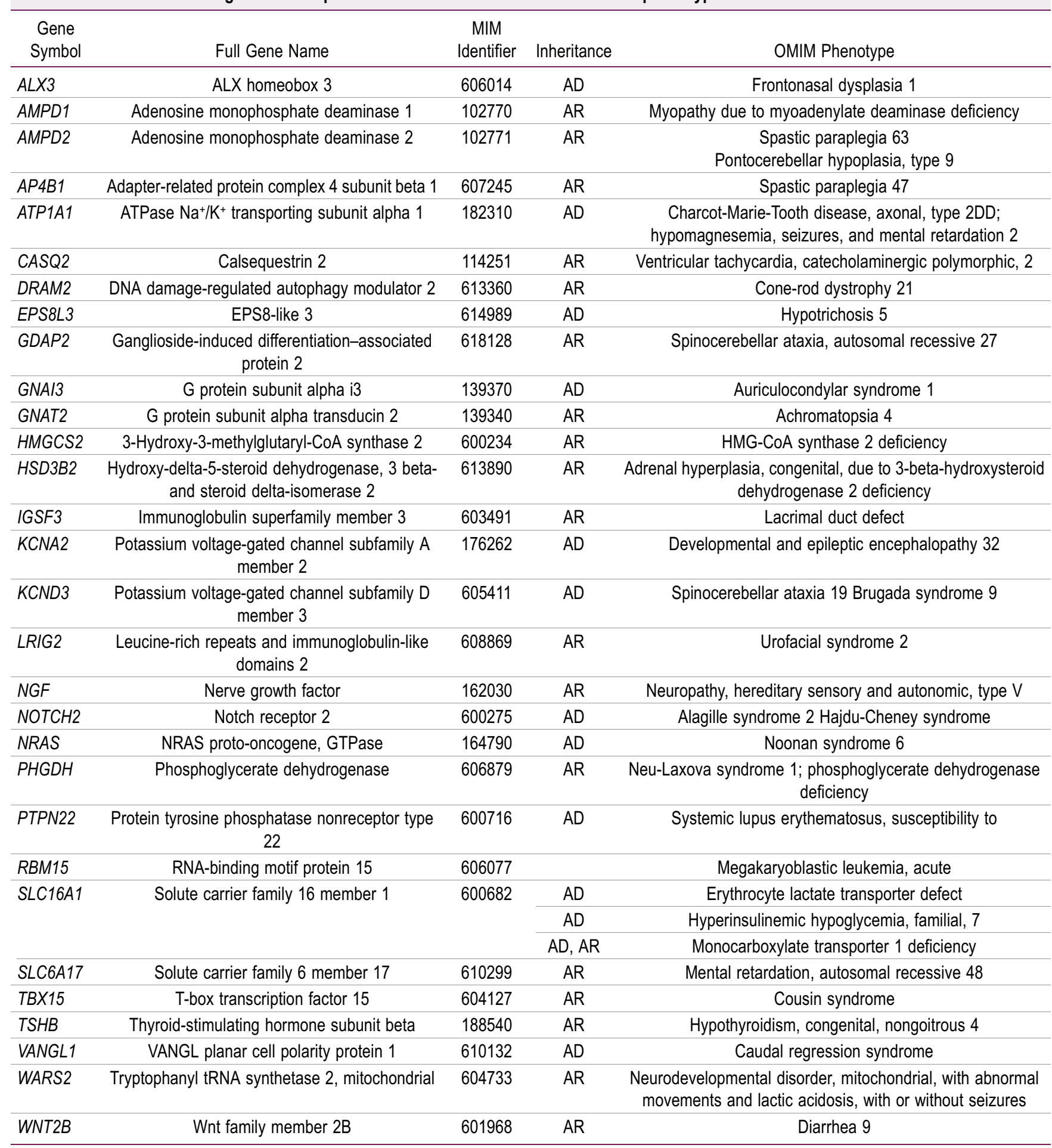

$A D$ = autosomal dominant; $A R$ = autosomal recessive; $C O A=$ coenzyme $A ; H M G$ = hydroxymethylglutaryl; OMIM = Online Mendelian Inheritance in Man.

compartments in the coronal suture. ${ }^{15}$ The missing NOTCH2 might be one of the factors linked to the particular fused squamosal suture in our reported case. However, craniosynostosis has not been reported in patients with the chromosome deletions similar to those in our patient. Linhares et al. reviewed patients with deletion of chromosome 1p13.2 and adjacent regions and concluded that NRAS gene haploinsufficiency caused clinical features overlapping Noonan syndrome. ${ }^{16}$ Our patient did not have facial features of Noonan syndrome, and her cardiac anomalies were not typical. Another possibility is a causative gene residing outside the deleted chromosome region. 


\section{Lessons}

Our case revealed that an isolated squamosal suture synostosis could be an uncommon feature of chromosome 1p12-1p13.3 deletion. Further study such as whole-exome/whole-genome sequencing may reveal a pathogenic variant in the gene causing craniosynostosis.

\section{References}

1. Ranger A, Chaudhary N, Matic D. Craniosynostosis involving the squamous temporal sutures: a rare and possibly underreported etiology for cranial vault asymmetry. J Craniofac Surg. 2010; 21(5):1547-1550.

2. Idriz S, Patel JH, Ameli Renani S, et al. CT of normal developmental and variant anatomy of the pediatric skull: distinguishing trauma from normality. Radiographics. 2015;35(5):1585-1601.

3. Ruengdit S, Troy Case D, Mahakkanukrauh P. Cranial suture closure as an age indicator: a review. Forensic Sci Int. 2020;307: 110111.

4. Murphy BD, Ajabshir N, Altman N, et al. Squamosal suture synostosis: incidence, associations, and implications for treatment. J Craniofac Surg. 2017;28(5):1179-1184.

5. Chieffe D, Naffaa L, Doumit G. Squamous suture synostosis: a review with emphasis on cranial morphology and involvement of other cranial sutures. J Craniofac Surg. 2017;28(1):51-55.

6. Greene AK, Mulliken JB, Proctor MR, et al. Phenotypically unusual combined craniosynostoses: presentation and management. Plast Reconstr Surg. 2008;122(3):853-862.

7. Smartt JM Jr, Singh DJ, Reid RR, et al. Squamosal suture synostosis: a cause of atypical skull asymmetry. Plast Reconstr Surg. 2012;130(1):165-176.

8. Eley KA, Thomas GP, Sheerin F, et al. The significance of squamosal suture synostosis. J Craniofac Surg. 2016;27(6):1543-1549.

9. Diab J, Anderson PJ, Moore MH. Late presenting bilateral squamosal synostosis. Arch Craniofac Surg. 2020;21(2):106-108.

10. Doumit GD, Sidaoui J, Meisler E, et al. Squamosal suture craniosynostosis in Muenke syndrome. J Craniofac Surg. 2014;25(2):429-431.

11. Tandon $Y K$, Rubin M, Kahlifa M, et al. Bilateral squamosal suture synostosis: a rare form of isolated craniosynostosis in Crouzon syndrome. World J Radiol. 2014;6(7):507-510.
12. Runyan $\mathrm{CM}, \mathrm{Xu} \mathrm{W}$, Alperovich $\mathrm{M}$, et al. Minor suture fusion in syndromic craniosynostosis. Plast Reconstr Surg. 2017;140(3): $434 \mathrm{e}-445 \mathrm{e}$.

13. Twigg SR, Versnel SL, Nürnberg G, et al. Frontorhiny, a distinctive presentation of frontonasal dysplasia caused by recessive mutations in the ALX3 homeobox gene. Am J Hum Genet. 2009;84(5): 698-705.

14. Narro-Donate JM, Méndez-Román P, Huete-Allut A, et al. Anterior unilateral plagiocephaly in patient with Alagille syndrome: case report. World Neurosurg. 2018;114:37-42.

15. Yen HY, Ting MC, Maxson RE. Jagged1 functions downstream of Twist 1 in the specification of the coronal suture and the formation of a boundary between osteogenic and non-osteogenic cells. Dev Biol. 2010;347(2):258-270.

16. Linhares ND, Freire MC, Cardenas RG, etal. $1 \mathrm{p} 13.2$ deletion displays clinical features overlapping Noonan syndrome, likely related to NRAS gene haploinsufficiency. Genet Mol Biol. 2016;39(3):349-357.

\section{Disclosures}

The authors report no conflict of interest concerning the materials or methods used in this study or the findings specified in this paper.

\section{Author Contributions}

Conception and design: Chaisrisawadisuk, Vatanavicharn, Anderson. Acquisition of data: Chaisrisawadisuk, Vatanavicharn. Analysis and interpretation of data: Chaisrisawadisuk, Vatanavicharn, Praphanphoj, Anderson. Drafting the article: Chaisrisawadisuk, Anderson, Moore. Critically revising the article: Chaisrisawadisuk, Vatanavicharn, Anderson. Reviewed submitted version of manuscript: Chaisrisawadisuk, Vatanavicharn, Praphanphoj, Moore. Approved the final version of the manuscript on behalf of all authors: Chaisrisawadisuk. Administrative/technical/material support: Vatanavicharn, Praphanphoj. Study supervision: Anderson.

\section{Correspondence}

Sarut Chaisrisawadisuk: Faculty of Medicine Siriraj Hospital, Siriraj, Bangkok Noi, Bangkok, Thailand. sarut.cha@mahidol.ac.th. 